\title{
Использование двухзеркальных двумерных брэгговских резонаторов в полупроводниковых гетеролазерах
}

\author{
(C) В.Р. Барышев, Н.С. Гинзбург, В.Ю. Заславский, А.М. Малкин \\ Институт прикладной фризики Российской академии наук, \\ 603950 Нижний Новгород, Россия \\ E-mail: baryshev@appl.sci-nnov.ru
}

Поступила в Редакцию 24 апреля 2019 г.

В окончательной редакции 29 апреля 2019 г.

Принята к публикации 29 апреля 2019 г.

\begin{abstract}
Предложена схема двухзеркального двумерного брэгговского резонатора на основе структур планарной геометрии, которая типична для гетеролазеров на основе межзонных переходов и квантово-каскадных лазеров. Отличительной особенностью таких резонаторов является обеспечение связи между продольными и поперечными потоками энергии в двумерных брэгговских зеркалах. Описан спектр собственных мод. Показана возможность использования резонаторов данного типа для синхронизации излучения лазеров и лазерных решеток.
\end{abstract}

Ключевые слова: гетеролазер, одномодовая генерация, брэгговские структуры, распределенная обратная связь.

DOI: $10.21883 /$ FTP.2019.09.48119.02

\section{1. Введение}

Полупроводниковые гетеролазеры (включая квантовокаскадные лазеры) в настоящее время являются востребованными источниками когерентного излучения в оптическом и инфракрасном диапазонах длин волн. Высокие значения коэффициента усиления активной среды позволяют реализовать лазерную генерацию в отсутствие внешних резонаторов, что в сочетании с токовой накачкой обеспечивает компактность и высокие показатели тепловой эффективности таких лазеров. Мощные гетеролазеры представляют собой плоскослоистые полупроводниковые структуры с тонким в масштабе длины волны активным слоем. При этом длина и ширина активной области может достигать сотен и тысяч длин волн. В отсутствие эффективных механизмов пространственной синхронизации излучения большой размер активной зоны может приводить к фактически независимой генерации в различных областях структуры. В результате выходное излучение будет характеризоваться широким спектром и низкой направленностью [1].

Основным способом улучшения спектральных свойств излучения, а также реализации одномодовых режимов генерации является интеграция в лазерные структуры брэгговских решеток на основе периодической модуляции коэффициента преломления в совокупности с ограничением размеров активной области. При этом наряду с традиционными одномерными брэгговскими решетками исследуются их различные модификации с более сложным профилем модуляции, существенно зависящим от двух пространственных координат [2,3].

В данной работе с этой целью предлагается использовать двумерные брэгговские резонаторы, представляющие собой участки планарных диэлектрических волно- водов с двумерно-периодической „шахматной“ модуляцией коэффициента преломления. Указанная модуляция обеспечивает связь волновых потоков, распространяющихся во взаимно перпендикулярных направлениях, что позволяет синхронизировать излучение по всей площади активной среды. Таким образом, за счет использования двумерной распределенной обратной связи возможна реализация одномодовой узкополосной генерации при больших размерах активной зоны, типичной для мощных гетеролазеров, с соответствующей большой интегральной интенсивностью светового потока $[4,5]$.

В настоящей работе рассматривается дополнительная возможность, состоящая в использования двухзеркальных резонаторов, составленных из двумерных брэгговских зеркал, разделенных отрезком регулярного планарного волновода (рис. 1). Каждое из зеркал представляет собой прямоугольный участок планарного

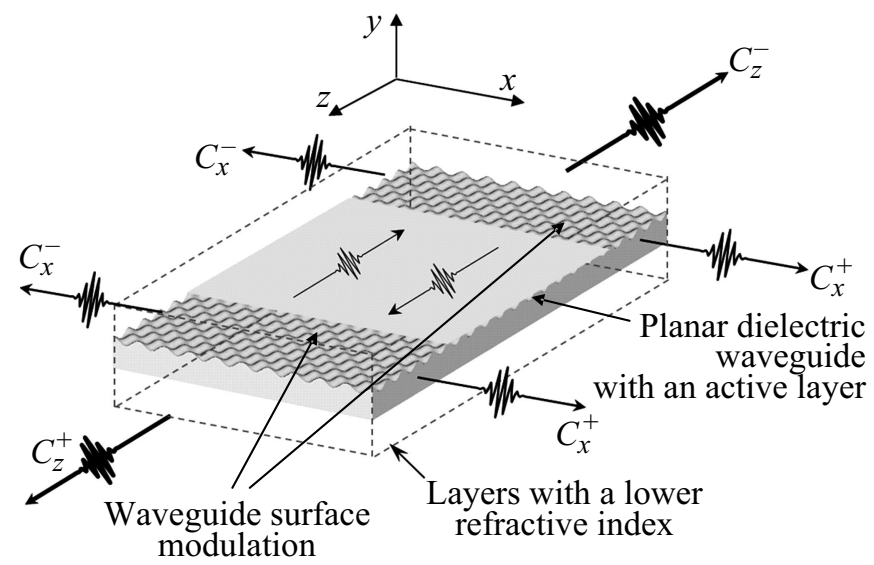

Рис. 1. Схема гетеролазера с двумерными брэгговскими зеркалами. 
диэлектрического волновода с шахматной модуляцией коэффициента преломления. Такие структуры подобны резонаторам Фабри-Перо и имеют аналогичный эквидистантный спектр высокодобротных мод. При этом наиболее добротная часть спектра мод ограничена полосой отражения брэгговских зеркал, определяемой глубиной модуляции. Как будет показано далее, предложенная схема позволяет обеспечить синхронизацию излучения протяженной в двух направлениях активной среды, что аналогично односекционным двумерным брэгговским резонаторам. В то же время двухзеркальный вариант отличается рядом дополнительных возможностей, из которых в работе будут подробно рассмотрены поперечный вывод излучения и многоканальная схема активной среды. В последнем варианте двумерные брэгговские зеркала фактически используются для синхронизации излучения лазерных решеток.

\section{2. Модель и основные уравнения}

Предлагаемая схема лазера с двумерными брэгговскими зеркалами изображена на рис. 1. Зеркала на основе двумерных брэгговских структур представляют собой участки планарных диэлектрических волноводов с двумерно-периодической гофрировкой поверхности синусоидальной формы:

$$
b(x, z)=b_{0}+b_{1,2}(\cos (\bar{h}(x+z))+\cos (\bar{h}(x-z))),
$$

где $b_{1,2}$ - амплитуды гофрировки в левом и правом зеркалах, $\bar{h}=2 \pi / d, d-$ период модуляции вдоль $x$ - и $z$-координат. Такие структуры при выполнении условий брэгговского резонанса:

$$
h \approx \bar{h}
$$

обеспечивают связь и взаимное рассеяние четырех волновых потоков, распространяющихся в ортогональных направлениях $[4,5]$ :

$$
\begin{aligned}
\stackrel{r}{A}=\operatorname{Re}[ & \left(\stackrel{r}{a}{ }_{1}(y)\left(C_{z}^{+} e^{-i h z}+C_{2}^{-} e^{i h z}\right)\right. \\
& \left.\left.+\stackrel{r}{a}_{2}(y)\left(C_{x}^{+} e^{-i h x}+C_{x}^{-} e^{i h x}\right)\right) e^{i \omega t}\right],
\end{aligned}
$$

где $\stackrel{r}{a}_{1,2}(y)$ описывают структуру распространяющихся в направлении $z$ и $x$ мод регулярного планарного диэлектрического волновода, $C_{z, x}^{+}(x, z, t)$ - медленно меняющиеся комплексные амплитуды парциальных волн. При этом волны $C_{z}^{+}$усиливаются в активной среде при распространении в регулярной области, а волны $C_{x}^{+}$ формируются за счет рассеяния излучения в зеркалах. Таким образом, считая структуру широкой в масштабе параметра Френеля $\left(l_{x}^{2} / l_{z} \lambda \gg 1\right)$ и пренебрегая дифракционным расплыванием, будем учитывать поперечные волновые потоки только внутри зеркал, т.е. $C_{x}^{+} \equiv 0$ при $z \notin\left(0, l_{1}\right)$ и $z \notin\left(l_{z}+l_{1} l_{z}+l_{1}+l_{2}\right)$. Здесь $l_{z}, l_{1}$ и $l_{2}$ - длины соответственно регулярной области, первого и второго брэгговских зеркал. Следует отметить, что при практической реализации, синусоидальная модуляция (1) может быть заменена шахматно-образной и может располагаться как на поверхности, так и внутри структуры на границе волноведущего слоя.

Взаимное рассеяние продольных и поперечных световых потоков описывается следующими уравнениями:

$$
\begin{aligned}
& \left( \pm \frac{\partial}{\partial Z}+\frac{\partial}{\partial \tau}\right) C_{z}^{+}+i \hat{\alpha}_{1,2}\left(C_{x}^{+}+C_{x}^{-}\right)=0, \\
& \left( \pm \frac{\partial}{\partial X}+\frac{\partial}{\partial \tau}\right) C_{x}^{+}+i \hat{\alpha}_{1,2}\left(C_{z}^{+}+C_{z}^{-}\right)=0,
\end{aligned}
$$

где $X=x / l_{1}, Z=z / l_{1}$ и $\tau=t v_{g} / l_{1}-$ нормированные пространственные координаты и время, $\hat{\alpha}_{1,2}=\alpha_{1,2} l_{1}-$ параметры связи волн для первого и второго зеркал. Выражения для параметров связи волн получены в работе [5].

Предположим, что активная среда расположена только в регулярной части резонатора. Поляризацию $P$ и $\rho$ инверсию активной среды представим в виде

$$
\begin{gathered}
P=\operatorname{Re}\left(i\left(P_{z}^{+} e^{i \bar{h} z}+P_{z}^{-} e^{-i \bar{h} z}\right) e^{i \omega_{0} t}\right), \\
\rho=\rho_{0}+\operatorname{Re}\left(\rho_{2 z} e^{2 i \bar{h} z}\right),
\end{gathered}
$$

где $\rho_{2 z}(x, z, t)$ - пространственная решетка инверсии, наведенная стоячей волной.

В рамках полуклассического подхода [6] процесс усиления может быть описан следующей системой уравнений в балансном приближении:

$$
\begin{gathered}
\left( \pm \frac{\partial}{\partial Z}+\frac{\partial}{\partial \tau}\right) \hat{C}_{z}^{ \pm}=\hat{P}_{z}^{ \pm}, \\
\frac{\partial \hat{\rho}_{0}}{\partial \tau}+\frac{\left(\hat{\rho}_{0}-1\right)}{\hat{T}_{1}}=-\operatorname{Re}\left(\hat{C}_{z}^{+} \hat{P}_{z}^{+*}+\hat{C}_{z}^{-} \hat{P}_{z}^{-*}\right), \\
\frac{\partial \hat{\rho}_{2 z}}{\partial \tau}+\frac{\hat{\rho}_{2 z}}{\hat{T}_{1}}=-\left(\hat{C}_{z}^{+} \hat{P}_{z}^{-*}+\hat{C}_{z}^{-} \hat{P}_{z}^{+*}\right), \\
\hat{P}_{z}^{+}=\beta \hat{T}_{2}\left(2 \hat{C}_{z}^{+} \hat{\rho}_{0}+\hat{C}_{z}^{-} \hat{\rho}_{2 z}\right), \\
\hat{P}_{z}^{-}=\beta \hat{T}_{2}\left(2 \hat{C}_{z}^{-} \hat{\rho}_{0}+\hat{C}_{z}^{+} \hat{\rho}_{2 z}^{*}\right) .
\end{gathered}
$$

Здесь используются следующие нормированные переменные:

$$
\begin{gathered}
\hat{\rho}=\frac{\rho}{\rho_{e}}, \hat{P}_{z}^{ \pm}=P_{z}^{ \pm}\left(\frac{\pi b_{\mathrm{eff}} l_{1}}{\rho_{e} h \omega_{0} c v_{g r} b_{\rho}}\right)^{1 / 2}, \\
\hat{T}_{1,2}=\frac{v_{g r} T_{1,2}}{l_{1}}, \beta=\pi \rho_{e}|\mu|^{2} b_{\rho} l_{1} c / 2 h \omega_{0} b_{\mathrm{eff}},
\end{gathered}
$$

где $\rho_{e}-$ равновесная концентрация активных элементов в отсутствие излучения, $\mu$ - дипольный момент, $T_{1,2}-$ времена релаксации инверсии и поляризации активной среды, $b_{\text {eff }}$ - эффективная толщина волновода для ТМ волн (см. [5]), $v_{g r}-$ групповая скорость волновых потоков, $b_{\rho}$ - толщина активного слоя. Размеры структуры нормированы аналогично координатам: $L_{2}=l_{2} / l_{1}$, $L_{z}=l_{z} / l_{1}$. 

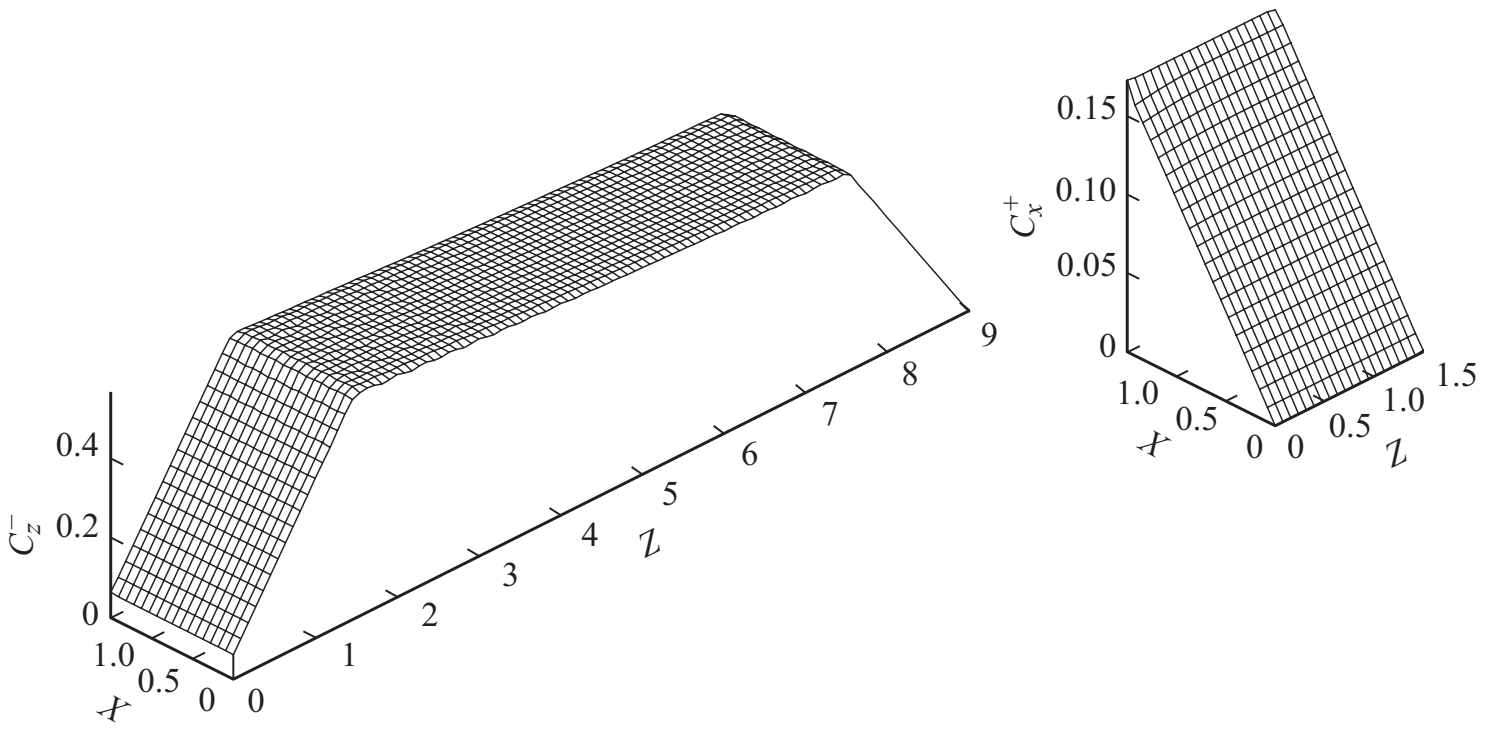

Рис. 2. Пространственная структура полей парциальных волн в стационарном режиме генерации; $L_{x}=1.5, L_{1}=L_{2}=1.5, L=6$, $\alpha=2, \gamma=0.033$.

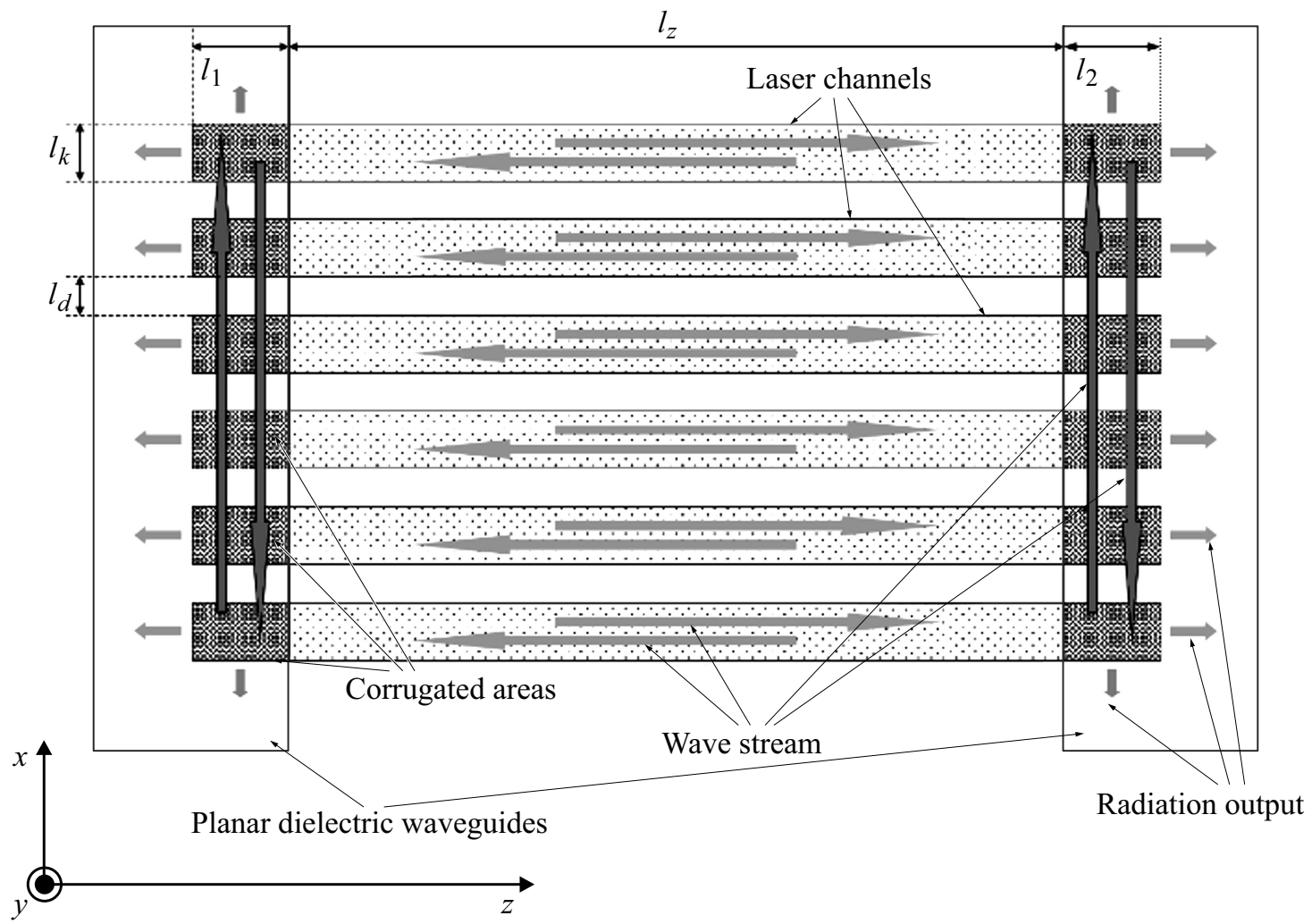

Рис. 3. Схема многоканального лазера с двумерными брэгговскими зеркалами.

\section{3. Модель лазера с вертикальным выводом излучения}

Одним из достоинств двухзеркальной схемы лазера с двумерными брэгговскими зеркалами является возможность вывода излучения в поперечном по отношению к плоскости волновода направлении. Вертикальный вывод излучения может быть реализован за счет модуляции поверхности волновода в регулярной области между зеркалами в виде

$$
b(z)=b_{0}+b_{v} \cos (\bar{h} z) .
$$

На такой модуляции парциальные волны $C_{z}^{ \pm}$рассеиваются в направлении $y$. Важно подчеркнуть, что непосред- 


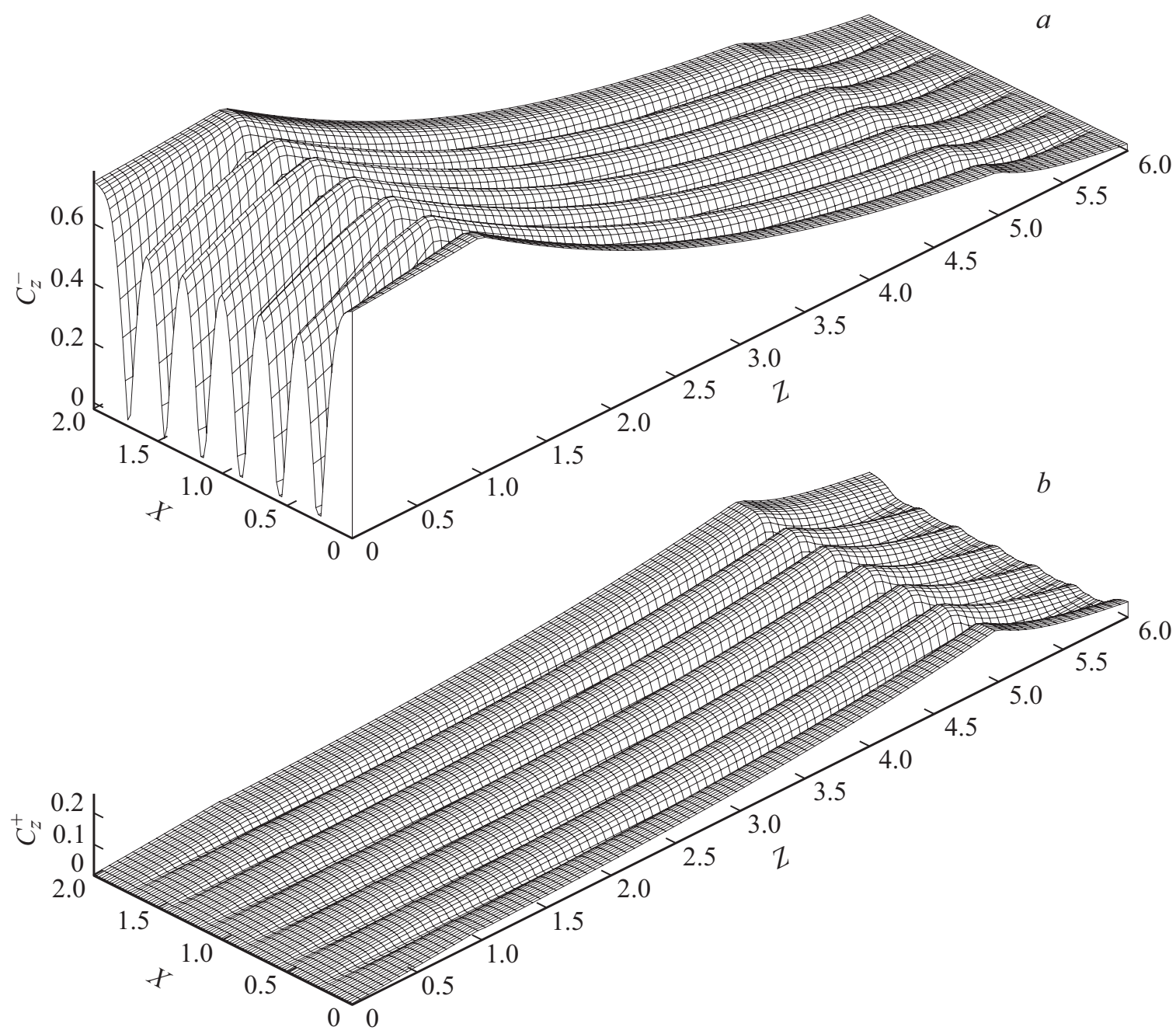

Рис. 4. Пространственная структура полей парциальных волн $C_{z}^{-}(a)$ и $C_{z}^{+}(b)$ в многоканальном лазере в стационарном режиме генерации; $L=4, L_{x}=2, L_{1}=L_{2}=1, \alpha_{1}=0.2, \hat{\alpha}_{2}=2, \beta=0.25$.

ственная связь встречных волн $C_{z}^{+}$и $C_{z}^{-}$отсутствует, поскольку для выполнения соответствующего брэгговского условия требуется в 2 раза меньший период модуляции. С точки зрения системы уравнений (6) резонансное рассеяние парциальных волн на решетке (7) аналогично введению эффективных омических потерь

$$
\left( \pm \frac{\partial}{\partial Z}+\frac{\partial}{\partial \tau}\right) \hat{C}_{z}^{ \pm}+\gamma \hat{C}_{z}^{ \pm}=\hat{P}_{z}^{ \pm} .
$$

Здесь коэффициент поперечного рассеяния $\gamma$ может быть получен, следуя процедуре, описанной в [7].

Результаты моделирования процесса генерации на основе уравнений (4) и (6) представлены на рис. 2. $\mathrm{B}$ некотором диапазоне значений параметра усиления $\beta$ реализуются стационарные одномодовые режимы генерации. При этом формально вывод излучения осуществляется в нескольких направлениях, но за счет подбора параметров двумерных брэгговских зеркал оказывается возможным обеспечить вывод подавляющей части светового потока в направлении, перпендикулярном плоскости волновода.

\section{4. Модель многоканального лазера с двумерными брэгговскими зеркалами}

Использование многоканальных схем в настоящее время является распространенным методом создания лазеров с большим сечением выходного пучка. Для когерентного сложения мощностей отдельных каналов необходима разработка методов синхронизации. Среди известных методов можно выделить использование внешних резонаторов [8], взаимную синхронизацию каналов за счет эффекта Тальбота [9], а также различные способы попарной связи каналов [10]. В настоящей работе применительно к инжекционным лазерам предлагается альтернативный вариант синхронизации лазерных каналов на основе двумерных брэгговских зеркал. Такая схема эффективна при планарной компоновке каналов и также позволяет совместить области распространения излучения с лазерными каналами.

Предлагаемая схема многоканального лазера изображена на рис. 3. Отдельные каналы в количестве $n$, дли- 
ны $l_{z}$ и ширины $l_{k}$ отстоят друг от друга на расстоянии $l_{d}$. Каналы связаны через специальные зеркала, представляющие собой планарные диэлектрические волноводы, на которые на определенных участках нанесены двумерные брэгговские структуры (1), связывающие продольные (ось $Z$ ) и поперечные (ось $X$ ) потоки энергии. В результате, как показано далее, удается осуществить взаимную синхронизацию излучения отдельных лазерных каналов.

Процесс генерации излучения в многоканальном лазере с двумерными брэгговскими зеркалами можно описать системой уравнений (4) и (6). Численное исследование в рамках описанной модели позволяет найти область значений тока накачки (параметр $\beta$ в уравнениях (6)), в которой реализуются стационарные режимы генерации с синхронизацией излучения отдельных каналов.

Важно подчеркнуть, что предполагается отсутствие существенных отражений от границ брэгговских структур, т. е. за границами гофрированных участков предполагается продолжение регулярного планарного диэлектрического волновода. Таким образом, генерируемое излучение выводится парциальными волнами $C_{x, z}^{ \pm}$в четырех направлениях. Однако распределение мощности излучения по направлениям может быть сделано существенно неравномерным за счет соответствующего выбора размеров и глубины гофрировки отдельно левого и правого зеркал. На рис. 4 представлены стационарные распределения амплитуд парциальных волн для случая, когда примерно 80\% мощности излучается через поверхность $Z=0$ парциальной волной $C_{z}^{-}$.

\section{5. Заключение}

При использовании в качестве внешних зеркал двумерные брэгговские структуры позволяют обеспечить синхронизацию излучения полупроводниковых лазеров. Спектр мод двухзеркального двумерного брэгговского резонатора расположен внутри полосы отражения зеркал, которая в свою очередь задается глубиной брэгговской модуляции. При этом коэффициенты отражения зеркал пропорциональны как глубине модуляции, так и площади зеркал, что дает некоторую свободу в независимом выборе указанных параметров. Наиболее добротная часть спектра представляет собой набор продольных мод, аналогичных модам резонатора Фабри-Перо. Как и в случае резонаторов типа Фабри-Перо с одномерными брэгговскими зеркалами, расположение продольных мод и центра полосы брэгговского отражения определяется взаимной фазировкой правого и левого зеркал. При этом точное совпадение центра полосы с продольной модой реализуется в случае синфазных решеток. Отметим, что для такого совмещения в резонаторе с однопериодическими брэгговскими зеркалами требуется скачок фазы.

Указанные особенности двухзеркальных двумерных брэгговских резонаторов позволяют использовать их для синхронизации излучения в полупроводниковых лазерных структурах, включающих как гетеролазеры на межзонных переходах, так и квантово-каскадные лазеры, а также лазерные решетки. При этом использование поперечных потоков энергии делает возможной реализацию одномодовых режимов генерации при размерах активной среды, многократно превышающих длину волны излучения. В то же время свобода в выборе параметров зеркал оказывается достаточной для реализации однонаправленного вывода излучения.

Отметим также, что рассматриваемая схема лазера с двумерными брэгговскими зеркалами представляется перспективной для синхронизации излучения твердотельных активных сред на основе редкоземельных элементов. Узкая спектральная полоса усиления таких сред могла бы существенно осложнить их использование в лазерах с односекционными двумерными брэгговских структурами ввиду необходимости попадания в узкую полосу усиления брэгговской частоты решетки. В двухзеркальном резонаторе за счет выбора параметров зеркал можно гарантировать попадание в полосу усиления одной из высокодобротных продольных мод резонатора и таким образом обеспечить возможность одномодовой генерации.

\section{Финансирование работы}

Работа выполнена в рамках государственного задания Института прикладной физики РАН на проведение научных исследований по теме № 0035-2014-013 и проекта Российского фонда фундаментальных исследований № 18-48-520022.

\section{Конфликт интересов}

Авторы заявляют, что у них нет конфликта интересов.

\section{Список литературы}

[1] А.П. Богатов, А.Е. Дракин, А.А. Стратонников, В. П. Коняев. Квант. электрон., 30 (5), 401 (2000).

[2] X. Sun, A. Yariv. Opt. Express, 16 (12), 9155 (2008).

[3] D.A. Yanson, E.U. Rafailov, G.S. Sokolovskii, V.I. Kuchinskii, A.C. Bryce, J. Marsh, W. Sibbett. J. Appl. Phys., 95, 1502 (2004).

[4] В.Р. Барышев, Н.С. Гинзбург, В.Ю. Заславский, А.М. Малкин, А.С. Сергеев, М. Тумм. Квант. электрон., 39, 463 (2009).

[5] N.S. Ginzburg, V.R. Baryshev, A.S. Sergeev, A.M. Malkin. Phys. Rev. A, 91, 053806 (2015).

[6] A.V. Andreev. Sov. Phys. Usp., 33 (12), 997 (1990).

[7] H. Kogelnik, C.V. Shank. Appl. Phys. Lett., 18 (4), 152 (1971).

[8] B. Liu, Y. Braiman, N. Nair, Y. Lu, Y. Guo, P. Colet, M. Wardlaw. Optics Commun., 324, 301 (2014).

[9] I.S. Goldobin. Sov. J. Quant. Electron., 19 (10), 1261 (1989).

[10] S. Peleš, J.L. Rogers, K. Wiesenfeld. Phys. Rev. E, 73, 026212 (2006).

Редактор Г.А. Оганесян 


\section{Semiconductor heterolasers with 2D Bragg resonators}

V.R.Baryshev, N.S. Ginzburg, V.Yu. Zaslavsky, A.M. Malkin

Institute of Applied Physics

of the Russian Academy of Sciences,

603950 Nizhny Novgorod, Russia

Abstract We suggested a two mirror 2D Bragg resonator scheme based on planar architecture, which is typical for both interband heterolasers and quantum cascade lasers. The distinguishing feature of those resonators is coupling between longitudinal and transverse energy streams in 2D Bragg mirrors. Eigenmode spectrum is described. Possibility of synchronizing laser radiation and laser arrays with two mirror 2D Bragg resonators is demonstrated. 